\title{
Inter-Dot Coupling in a Self-Assembled Quantum Dot System
}

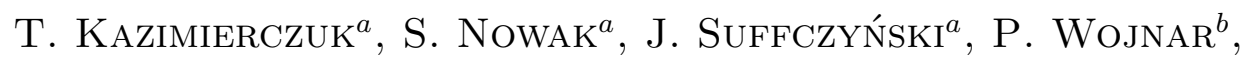 \\ A. GOLnIK ${ }^{a}$, J.A. GAJ ${ }^{a}$ AND P. KOSSACKI ${ }^{a}$ \\ ${ }^{a}$ Institute of Experimental Physics, Warsaw University \\ Hoża 69, 00-681 Warsaw, Poland \\ ${ }^{b}$ Institute of Physics, Polish Academy of Sciences \\ al. Lotników 32/46, 02-668 Warsaw, Poland
}

We present studies of resonant excitation of self-assembled $\mathrm{CdTe} / \mathrm{ZnTe}$ quantum dots. Photoluminescence excitation measurements revealed existence of sharp resonances, common for photoluminescence lines attributed to different quantum dot charge states. We conclude from the ensemble of photoluminescence and photoluminescence excitation results that we observe energy transfer in coupled quantum dot pairs.

PACS numbers: 78.67.Hc, 78.55.Et

\section{Introduction}

Semiconductor quantum dots (QDs) attract attention as non-classical light sources and potential systems for quantum information processing. Unless special efforts are undertaken to produce vertically or horizontally coupled pairs, self-assembled QDs are usually considered to be separated one from another. Our study shows that under certain condition inter-dot interactions can be pronounced even in a single layer self-assembled system.

\section{Samples and experiment}

The experiments were carried out on a sample containing a single layer of self-assembled CdTe/ZnTe QDs. The formation of the QDs was induced by introducing a layer of amorphous tellurium which was later desorbed [1]. QD layer was formed from 6 monolayers of CdTe and average QD density was estimated to be $10^{10} \mathrm{~cm}^{-2}$ from atomic force microscopy (AFM) experiments on similar samples.

The experiments were performed in a microphotoluminescence setup. The sample was mounted on a microscope objective [2] and placed inside a cryostat at 
$T=1.6 \mathrm{~K}$. QDs were excited by a CW dye laser with Rhodamine $6 \mathrm{G}$, assuring a tuning range of approximately $2090-2140 \mathrm{meV}$, which is lower than an energy gap of ZnTe barriers. $\lambda / 2$ waveplate was used to rotate the polarization axis of the exciting light while a polarizer in front of monochromator allowed us to select photoluminescence (PL) of given polarization.

Identification of optical transitions in the PL spectrum was based on relative energy positions and polarization properties as well as on photon correlation measurements performed on individual QD PL lines. Hanbury-Brown and Twiss correlation setup with avalanche photodiodes was used. Its detailed description is given in [3].

\section{Results and discussion}

Figure 1 shows example photoluminescence and photoluminescence excitation (PLE) spectra obtained on a typical quantum dot. Photoluminescence spectrum of a single QD (Fig. 1b) consists of a set of sharp lines. We identified the lines related to recombination of neutral exciton (X), biexciton (XX), charged exciton (CX), and charged biexciton (CXX). The preliminary correlation results indicate that the line denoted as UX is related to trion of the charge opposite to that of CX but the identity of the line is not important for the present work. As shown in Fig. 1b, the sharp resonances in PLE spectra are observed. Their energies are not correlated to the emission energy of the dots. Moreover, the intensities of all PL lines of single QD are enhanced at the same resonance (Fig. 1b). The width of the resonance lines is comparable to the width of the excitonic emission lines from QDs. We use a polarization anisotropy analysis in order to determine the origin of the resonances.

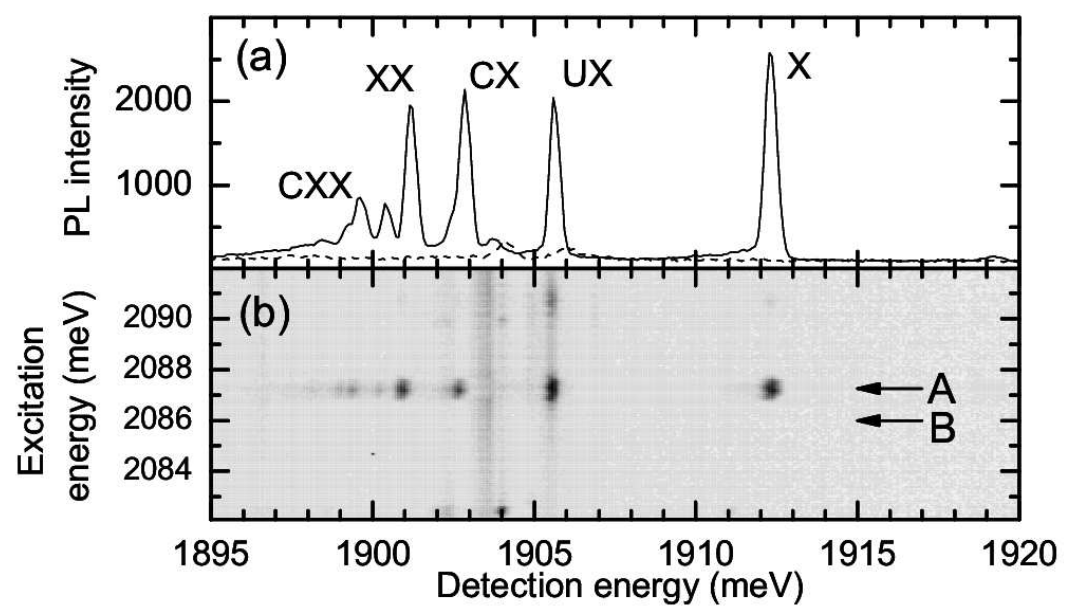

Fig. 1. PL spectra excited in the resonance (solid line - A) and $1 \mathrm{meV}$ detuned from resonance (dashed line - B) are presented in (a). PLE measured on a single QD (b). 
It is well established that quantum dots often exhibit in-plane anisotropy. In the case of II-VI compounds it was proven that principal axes of the QDs are randomly oriented and are not related to crystallographic axes [4]. The anisotropy is visible in the PL spectrum as splitting of the lines X and XX into pairs of linearly polarized components. In the experiment we measured PL spectrum as a function of angle of luminescence light polarization. In our case the splitting was smaller than the line width, therefore oscillations in intensity of two components were observed as oscillations of the spectral line position. The example results are presented in Fig. 2a. As expected, X and XX lines exhibit energy oscillations that correspond to a splitting of $140 \mu \mathrm{eV}$. The principal axes of the QD were determined at $-23^{\circ}$ and $67^{\circ}$ with respect to the cleaving direction (110).

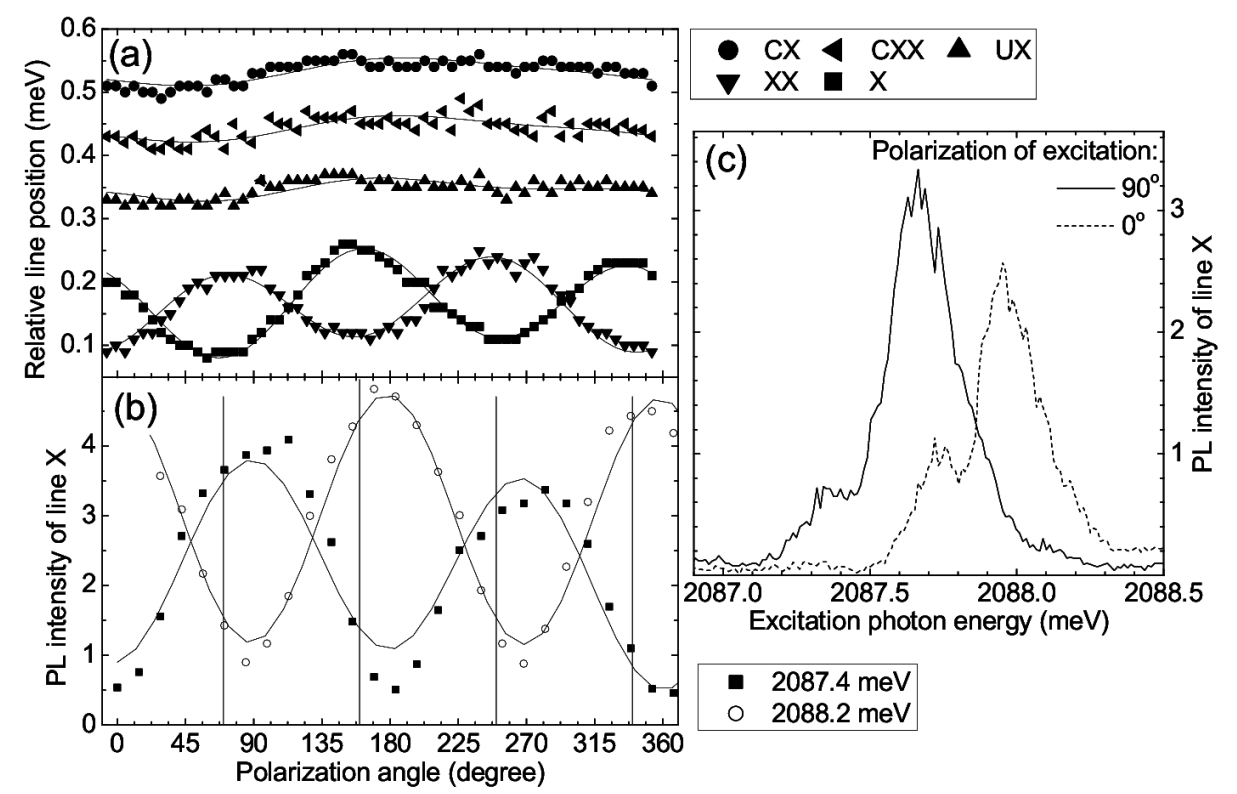

Fig. 2. Example results of the anisotropy analysis for emission (a) and excitation lines (b). Vertical lines mark principal axes of the dot deduced from the $\mathrm{X}$ emission anisotropy. Part (c) shows a PLE spectrum of the QD measured for X line (let us note the energy scale).

A detailed investigation revealed that the resonances in PLE spectra consist of two linearly polarized components (Fig. 2c). An exact orientation of the polarization axes of two components was determined by tuning the laser to one of the components of the resonant peak and rotating the polarization of the exciting light. In the case of the dot described previously we determined the values of $-4^{\circ}$ and $86^{\circ}$, which are different from the orientation of the principal axes of PL. The analysis of several such resonances for different quantum dots revealed no correlation between orientation of PLE principal axes, PL principal axes, and crystallographic axes. 
We explain the observed finding as due to coupling between two quantum dots with different principal axes. The proposed interpretation is supported by the fact that the distance between two components of PLE spectrum is of the order of magnitude of anisotropic splitting of exciton state in QDs. Moreover, the participation of another quantum dot in the excitation process explains why the observed resonance is common for neutral (X, XX) and charged (CX, CXX) states of quantum dot.

\section{Conclusions}

We found sharp resonances in photoluminescence excitation spectra of individual QDs in a single layer self-assembled system. The resonances exhibit optical anisotropy oriented differently than that of QD photoluminescence. The resonances have a form of linearly polarized doublets. They occur simultaneously for all principal QD PL lines attributed to different charge states. We conclude that the observed effects originate from coupled QD pairs in the self-assembled system.

\section{Acknowledgments}

This work was partially supported by the Polish Ministry of Science and Higher Education as research grants in years 2006-2010.

\section{References}

[1] F. Tinjod, B. Gilles, S. Moehl, K. Kheng, H. Mariette, Appl. Phys. Lett. 82, 4340 (2003).

[2] J. Jasny, J. Sepiol, T. Imgartinger, M. Trauber, A. Renn, U.P. Wild, Rev. Sci. Instrum. 67, 1425 (1996).

[3] J. Suffczyński, T. Kazimierczuk, M. Goryca, B. Piechal, A. Trajnerowicz, K. Kowalik, P. Kossacki, A. Golnik, K.P. Korona, M. Nawrocki, J.A. Gaj, G. Karczewski, Phys. Rev. B 74, 085319 (2006).

[4] A. Kudelski, A. Golnik, J.A. Gaj, S. Maćkowski, G. Karczewski, J. Kossut, in: Proc. 25th Int. Conf. on the Physics of Semiconductors, Osaka 2000, Eds. N. Miura, T. Ando, in series Springer Proceedings in Physics, Vol. 87, Springer, Berlin 2001, p. 1249. 